\title{
Pre and Post-Natal Imaging of a Patient with Dandy- Beaumont Walker Malformation in Combination with Pontine Tegmental Cap Dysplasia: Novel Case and Possible Etiology
}

Ryan Kelsch, $\mathrm{DO}^{1}$; Luis Goncalves, $\mathrm{MD}^{2}$; Anant Krishnan, $\mathrm{MD}^{1}$

${ }^{1}$ Department of Radiology and Molecular Imaging, Beaumont Hospital, Royal Oak, MI - Department of Radiology and Imaging, Pheonix Children's, Phoenix, AZ

\section{Purpose}

The purpose of this presentation is to present a novel case of Dandy-Walker malformation in combination with pontine tegmental cap dysplasia. This case will be used to illustrate the common imaging findings for each disease entity and how they might relate in etiology. Multimodality pre-natal and post-natal imaging will both be reviewed.

\section{Case Report}

The mother of the patient presented to our institution as a 35 year old G4P2012 for concerning findings on fetal ultrasound. In-utero the patient was suspected to have Dandy-Walker malformation via ultrasound and then underwent a fetal MRI. The patient was followed with serial ultrasounds and had a subsequent delivery via cesarean section. The patient was found to have multiple congenital abnormalities with the VACTERL association. Neurologically the patient was found to have bilateral sensorineural hearing loss, feeding difficulties, and partial symptomatic epilepsy with complex partial seizures. The patient was managed with a ventriculoperitoneal shunt and antiepileptic medications, and is being followed by neurology and neurosurgery.

Figure 1. Figure 1.A, 29 week gestation prenatal US imaging demonstrating large posterior fossa cystic lesion (blue arrow). B, 30 week gestation sagittal SS-FSE, C, 2 day old sagittal MPRAGE, D, 11 month old head ultrasound, and E, 12 month old sagittal MPRAGE imaging demonstrating posterior deformity of the pons (green arrow), large posterior fossa cyst (blue arrow), and severely hypoplastic cerebellar vermis (red arrow).
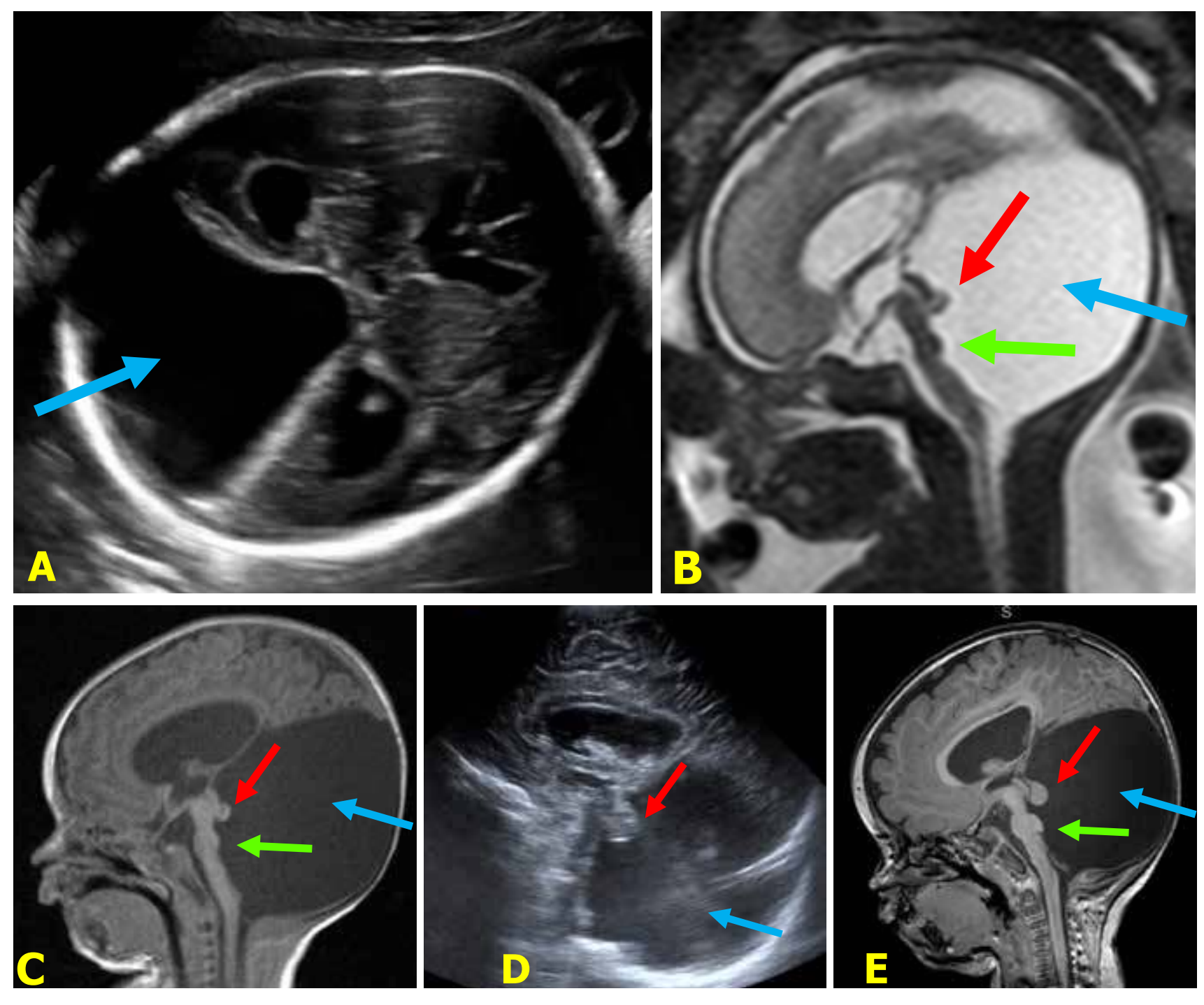

Imaging

PRENATAL US, 29 WEEKS GESTATION (Figure 1A): Demonstrated a large posterior fossa cystic space with hypoplastic cerebellar hemispheres, ventriculomegaly, rib fusions, penile abnormality, and no anal dimple.

PRENATAL MRI, 30 WEEKS GESTATION (Figure 1B): Demonstrated a large posterior fossa cyst, elevation of the torcula, a severely hypoplastic vermis, a hypoplastic pons with posterior tegmental fullness, ventriculomegaly, partial absence of cavum septum pallucidum, and a high anorectal malformation. Hypospadias, lumbar spine segmentation abnormalities, and rib fusion abnormalities were also seen. POSTNATAL MRI, 2 DAYS OLD (Figure 1C):

Demonstrated a malrotated and severely hypoplastic cerebellar vermis, cystic enlargement of the posterior fossa, torcular inversion, and posterior deformity of pons consistent with tegmental cap dysplasia.

POSTNATAL HEAD US, 11 MONTHS OLD (Figure 1D): Demonstrated a posterior fossa cyst, hydrocephalus, and diminutive pons.

POSTNATAL MRI, 12 MONTHS OLD (Figure 1E): Demonstrated a malrotated and severely hypoplastic cerebellar vermis, cystic enlargement of the posterior fossa, torcular inversion, a posterior deformity of pons consistent with tegmental cap dysplasia, hypoplastic middle cerebral peduncles, and a suggestion of hypoplasia of cranial nerves VII/VIII with hypoplastic internal auditory canals.

\section{Summary}

Dandy-Walker malformation and pontine tegmental cap dysplasia (PTCD) are an unusual combination of pathologies. While vermian hypoplasia has been reported with PTCD, we are not aware of a Dandy walker malformation, as diagnosed by prenatal US or MRI, in combination with this condition. We suggest that both conditions may arise from a defect in cell migration and folding during the formation of the rhombencephalon. While it is unclear where the dysplastic tegmental cap axonal fibers originate form, perhaps this area of dysplastic tissue contains fibers from the middle cerebellar peduncle normally present within the vermis, decreasing its mass.

\section{DISCLOSURES}

Nothing to disclose.

\section{Reference}

